\title{
INFLUENCE OF SHIP MOTION NONLINEARITIES ON THE COURSE CONTROLLER DESIGN
}

\author{
Markus Graswald, Holger Korte, \\ Bernhard P. Lampe
}

\author{
Institute of Automation, University of Rostock, \\ Richard-Wagner-Straße 31, D-18119 Rostock, Germany \\ email: markus.graswald@uni-rostock.de
}

\begin{abstract}
Since a ship yard usually provides insufficient data for developing a ship control system, the course and track controller design is based upon a Nomoto model determined with a Clarke estimation of hydrodynamic derivatives. Several ships examined show a poor system performance with a course control derived from these models besides parameter identification problems of adaptive control concepts. Hence, the steering quality of the Mariner class is discussed and linear model parameters are obtained by an interval approximation of Dieudonné's spiral curve in the working range of the rudder. This approach leads to a better controlled nonlinear process. Copyright (C) 2005 IFAC
\end{abstract}

Keywords: Ship control, Manoeuvrability, Stability, Parameter estimation, Least-squares approximation

\section{INTRODUCTION}

In general, course and track controls of modern ships are widely designed on the basis of linear Nomoto models (Nomoto et al., 1957). Since only rudimentary information, e.g., main data, dimensions, and the proof of dynamic yaw stability, can be obtained during the control design process, the linear velocity and acceleration derivatives of the mathematical model are usually estimated by the hull geometry referring to (Clarke et al., 1982). Another approach for determining the hydrodynamic derivatives is to conduct experimental methods measuring forces and moments, especially for those new ship types equipped with azimuthing propulsors. Problems still exist also for older vessels in transferring these model-scale results to full-scale (Mewis, 2001; Kleinau, 1976).

These experiences can be confirmed for different types of ships in our institute showing that nonlin- earities of the plant are still problematic to handle. This may finally lead to inaccuracies in identifying mathematical models, inefficient controllers, and a higher rate of wear of the rudder engine (Korte et al., 2002).

Therefore, in the first part of this paper, identification results of a modern ro-ro ferry used for a maneuver prediction system are presented. In order to show the importance of an accurate hydrodynamic model and in absence of such a model for the investigated vessel, the second part consists of a controller design for the well examined Mariner class considering the nonlinear model and Dieudonné's spiral curve. The objective is to find Nomoto parameters depicting more realistically dynamic properties and to develop a PID-controller resulting in a better system performance after a course alteration using the nonlinear model of the vessel. 


\section{MANEUVER PREDICTION OF SHIPS}

The MAPSYS project ("Manoeuvre Prediction System for Ships") aimed at improving the nautical safety of ships in confined waters and harbor approaches with major current gradients by developing a prediction system using external current measurements (Korte et al., 2001). The vessel "Transeuropa", a ro-ro/passenger ferry of the Finnlines Group built in 1995, was examined during this project $\left(L_{p p}=171.3 \mathrm{~m}, \nabla=\right.$ $\left.11,646 \mathrm{DWT}, u_{0}=21 \mathrm{kts}\right)$. The prediction model consists of an adaptive linear time-variant dynamic model and a cascade control module with an inner course autopilot loop and an outer trajectory tracking control loop.

Describing the dynamics of the ship, a nonlinear a priori model is usually determined requiring many parameters obtained by measurements or design drawings. Furthermore, the hydrodynamic derivatives depend on the loading conditions and the surge velocity. Therefore, these models are inappropriate for practical purposes like a prediction system of ship motion or a control system design, since system identification with full-scale maneuvering trials is usually hindered due to economic and nautical constraints (Korte et al., 2002).

\subsection{Identification of a Linear Ship Model}

Due to the reasons mentioned, only a few zigzag and course alteration maneuvers can be performed. The gathered data is used for determining parameters of, at first, the Nomoto course model and, secondly, the track model applying the prediction-error identification method (PEM).

In order to obtain an overview, the parameters of Nomoto's 2nd-order model are estimated:

$$
G_{\psi}(s)=\frac{K_{I S}}{s} \cdot \frac{1}{\left(1+T_{S} s\right)},
$$

where $K_{I S}$ - gain and $T_{S}=T_{1}+T_{2}-T_{3}-$ Nomoto's time constant.

This is not trivial because the measured signals are disturbed and the number of estimated parameters is additionally increased due to initial states, i.e., the rudder bias $\delta_{R 0}$, the course angle $\psi_{0}$, and yaw rate $r_{0}$.

The analysis of an automatic course alteration maneuver and a helmsman-steered $5^{\circ}-5^{\circ}$ maneuver at $u=20 \mathrm{kts}$ show both a good approximation of the measured and the simulated course angle, see Fig. 1 and 2. Comparing the identified Nomoto parameters $K_{I S}$ and $T_{S}$ to those of the "Montebello", another ship with a similar size and design speed examined during the former INISproject (Lampe et al., 1998), similar results should
Table 1. Identified Parameters of the Course Alteration Maneuver.

\begin{tabular}{lcc} 
& Initial values & Estimated parameters \\
\hline$K_{I S}$ in s & 0.06 & 0.1003 \\
$T_{S}$ in s & 20.0 & 43.8563 \\
$\delta_{R 0}$ in & & -1.3766 \\
$r_{0}$ in $^{\circ} \mathrm{s}^{-1}$ & 0.0 & -0.0304 \\
$\psi_{0}$ in $^{\circ}$ & 0.0 & 0.1764 \\
\hline
\end{tabular}

Table 2. Identified Parameters of the Manually Steered Zig-Zag Maneuver.

\begin{tabular}{lcc} 
& Initial values & Estimated parameters \\
\hline$K_{I S}$ in s & 0.06 & 0.2318 \\
$T_{S}$ in s & 20.0 & 93.7038 \\
$\delta_{R 0}$ in ${ }^{\circ}$ & 0.0 & -1.1817 \\
$r_{0}$ in $^{\circ} \mathrm{s}^{-1}$ & 0.0 & 0.2142 \\
$\psi_{0}$ in $^{\circ}$ & 0.0 & -3.2598 \\
\hline
\end{tabular}

be gained applying fundamental laws of physics. However, the calculated quantities are strongly different and partially unrealistic, Table 1 and 2 . The approach of identifying the parameters in two steps, which can be repeated in order to obtain more converged results, leads to expected values. For this case, the simulation provides unsatisfactory results.

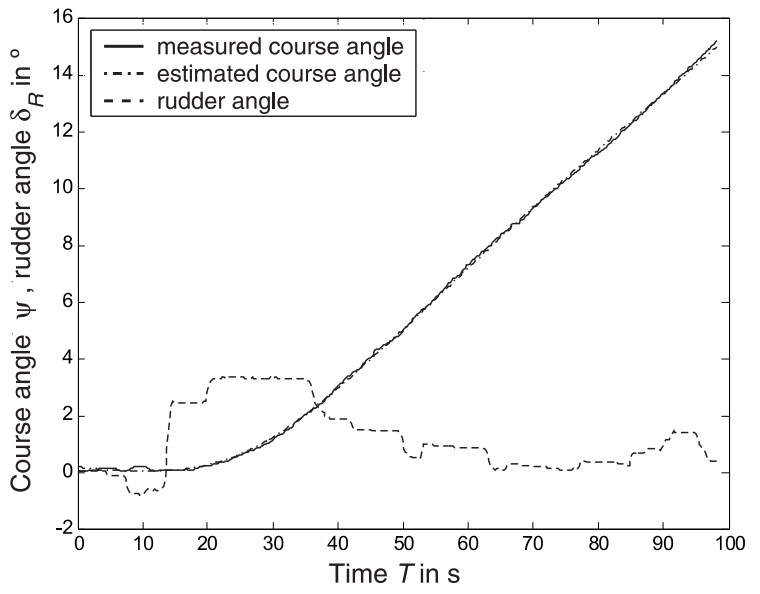

Fig. 1. Automatically Driven Course Alteration Maneuver without Transient.

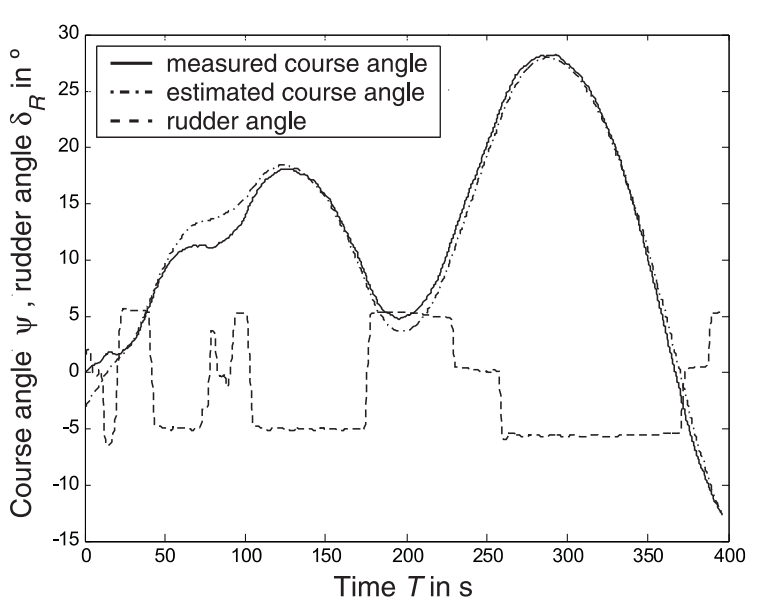

Fig. 2. Manually Steered $5^{\circ}-5^{\circ}$ Maneuver.

Therefore, the Nomoto model cannot reproduce the true dynamics of this particular vessel. Al- 
though the condition of yaw stability is fulfilled for this ship, it is suspected that a nonlinearity occurring within a small rudder range causes this problem. It is indicated by major rudder movements compared to the rudder angle of the course alteration during a compensated straightline motion of the vessel at calm weather, Fig. 3 . The obvious wear of the rudder engine supposes that these difficulties have been already existed since implementing the control system at the yard. (Korte et al., 2002)

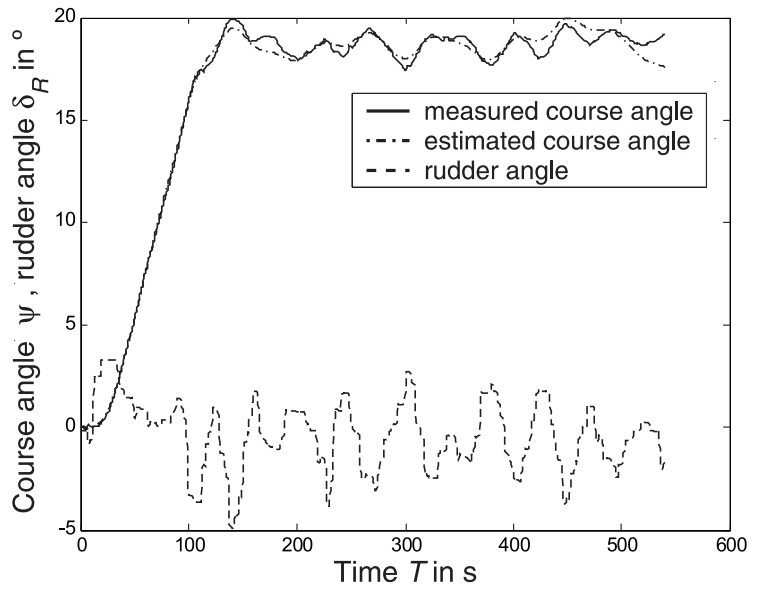

Fig. 3. Automatic Course Alteration Maneuver.

Similar observations were made for determining linear model parameters of type 301 "Pioner" built on the "Neptunwerft" shipyard Rostock (Kleinau, 1976) and the Mariner class being examined in the following section (Chislett and StrømTejsen, 1965).

\section{INVESTIGATION OF THE MARINER CLASS}

The known Mariner class $\left(L_{p p}=160.9 \mathrm{~m}, \nabla=\right.$ $16,622 \mathrm{DWT}, u_{0}=15 \mathrm{kts}$ ) is chosen for further investigations, since comprehensive Planar Motion Mechanism (PMM) tests, and full-scale steering and maneuvering predictions were performed in Lyngby, Denmark, as well as several applications in the field of modeling and control system design were published, e.g., (Fossen, 1994).

\subsection{Mathematical Model}

The nonlinear 3 DOF equations of motion in surge, sway, and yaw as derived by (Chislett and Strøm-Tejsen, 1965) assuming an undisturbed water surface are used as a reference model:

$$
\begin{aligned}
m_{x} \dot{u} & =X, \\
m_{y} \dot{v}+\left(m x_{G}-Y_{\dot{r}}\right) \dot{r} & =Y, \\
I_{z z} \dot{r}+\left(m x_{G}-N_{\dot{v}}\right) \dot{v} & =N,
\end{aligned}
$$

where

$$
\begin{aligned}
& X=X_{u} u+X_{u u} u^{2}+X_{u u u} u^{3}+X_{v v} v^{2}+X_{r r} r^{2} \\
&+X_{r v} r v+X_{\delta \delta} \delta^{2}+X_{u \delta \delta} u \delta^{2}+X_{v \delta} v \delta \\
&+X_{u v \delta} u v \delta \\
& Y=Y_{v} v+Y_{r} r+Y_{v v v} v^{3}+Y_{v v r} v^{2} r+Y_{v u} v u \\
&+Y_{r u} r u+Y_{\delta} \delta+Y_{\delta \delta \delta} \delta^{3}+Y_{u \delta} u \delta+Y_{u u \delta} u^{2} \delta \\
&+Y_{v \delta \delta} v \delta^{2}+Y_{v v \delta} v^{2} \delta+Y 0+Y 0_{u} u+Y 0_{u u} u^{2}, \\
& N=N_{v} v+N_{r} r+N_{v v v} v^{3}+N_{v v r} v^{2} r+N_{v u} v u \\
&+N_{r u} r u+N_{\delta} \delta+N_{\delta \delta \delta} \delta^{3}+N_{u \delta} u \delta+N_{u u \delta} u^{2} \delta \\
&+N_{v \delta \delta} v \delta^{2}+N_{v v \delta} v^{2} \delta+N 0+N 0_{u} u+N 0_{u u} u^{2}, \\
& \text { and } m_{x}=m-X_{\dot{u}}, m_{y}=m-Y_{\dot{v}}, I_{z z}=I_{z}-N_{\dot{r}} .
\end{aligned}
$$

Allowing only small perturbations from nominal values, i.e., $u \approx u_{0}=$ const. $\gg v, r$, and neglecting roll and heave, linear theory suggests the state-space model for the steering motion:

$$
\begin{aligned}
& \dot{\boldsymbol{x}}=\boldsymbol{A} \boldsymbol{x}+\boldsymbol{b} u, \\
& \boldsymbol{y}=\boldsymbol{C} \boldsymbol{x}+\boldsymbol{d} u,
\end{aligned}
$$

where $\boldsymbol{x}=\left[\begin{array}{lll}v & r & \psi\end{array}\right]^{T}, u=\delta=-\left(\delta_{R}+\delta_{R 0}\right), \boldsymbol{y}=\boldsymbol{x}$,

$$
\boldsymbol{A}=\left[\begin{array}{ccc}
a_{11} & a_{12} & 0 \\
a_{21} & a_{22} & 0 \\
0 & 1 & 0
\end{array}\right], \boldsymbol{b}=\left[\begin{array}{c}
b_{1} \\
b_{2} \\
0
\end{array}\right], \boldsymbol{C}=\boldsymbol{I}_{\mathbf{3} \times \mathbf{3}}, \boldsymbol{d}=\mathbf{0} .
$$

Laplace transformation of Eq. (3) and some rearrangements yields the transfer function of Nomoto's 3rd-order model:

$$
G_{\psi}(s)=\frac{\Psi(s)}{\Delta(s)}=\frac{K_{I S}}{s} \cdot \frac{1+T_{3} s}{\left(1+T_{1} s\right)\left(1+T_{2} s\right)},
$$

which can be simplified to the 2nd-order model, Eq. (1).

The derivation of the Nomoto model requires dynamic yaw stability. Referring to (Schmitz, 1961), a ship is dynamically stable, if disturbances of the drift angle or its angular velocity decay for a ship on a steady trajectory. It can be proved that if the real parts of the roots of the characteristic equation are negative, the system is stable. This criterion is fulfilled for a Mariner class vessel.

For the vessel given, the Nomoto parameters of the course plant are determined through linearization at system equilibrium, i.e., the nominal value is $\delta_{R c}=0^{\circ}$, and shown in Table 3 .

Neglecting the influence of the rudder rate limiter for design purposes, the dynamics of the rudder engine are approximated as a $\mathrm{PT}_{1}$-element (Mierau, 1983):

$$
G_{A}(s)=\frac{\Delta(s)}{\Delta_{c}(s)}=\frac{K_{A}}{1+T_{A} s},
$$

where $K_{A}=1 \mathrm{~s}^{-1}$ and $T_{A}=1 \mathrm{~s}$. 


\subsection{Analysis of the Turning Ability}

In this subsection, Dieudonné's spiral and its influence on the turning ability of a ship is discussed. The spiral curve provides a nonlinear relationship between constant (commanded) rudder angles $\delta_{R c}$ and steady-state yaw rates at a particular nominal surge velocity $u_{0}$.

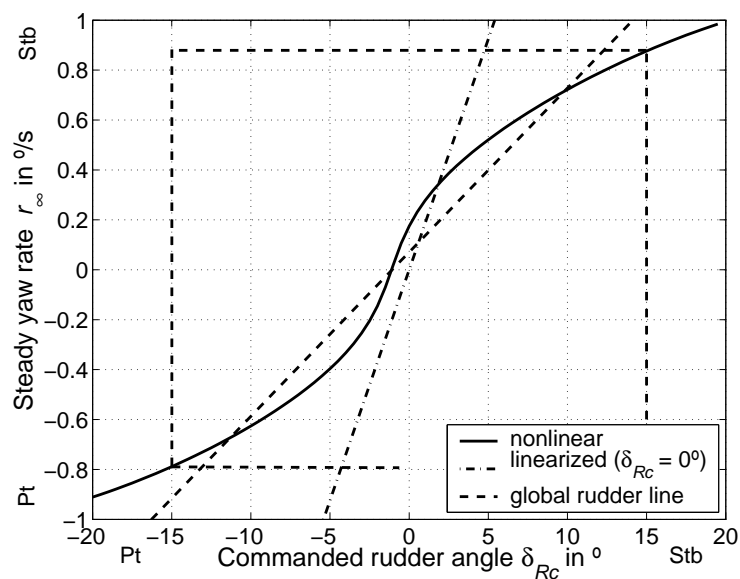

Fig. 4. $r-\delta$ Diagram, Nomoto's Characteristic Line, and Global Rudder Characteristic Line.

As depicted in Fig. 4, the spiral curve of the Mariner class can only be linearly approximated in a considerably small section, i.e., $-2^{\circ} \leq \delta_{R c} \leq$ $0^{\circ}$. It indicates that the conventional Nomoto model linearized at $\delta_{R c}=0^{\circ}$ cannot be used for a course controller design meeting sufficient dynamic results, because usually a larger rudder range is required. Due to the steering effect of the propeller, an offset of the origin $\delta_{R 0}=-1.11^{\circ}$ is caused, commonly referred to as rudder bias.

Therefore, a least-squares method is adopted in order to determine the linear regression of the spiral curve in the defined rudder range of interest (see dashed line box in Fig. 4). It is calculated as

$$
y_{i}=\hat{\beta_{0}}+\hat{\beta}_{1} x_{i} ; i=1, \ldots, N
$$

where the regression coefficients are

$$
\begin{aligned}
& \hat{\beta_{0}}=\frac{\sum_{i=1}^{N} y_{i}-\hat{\beta}_{1} \sum_{i=1}^{N} x_{i}}{N}, \\
& \hat{\beta_{1}}=\frac{N \sum_{i=1}^{N} x_{i} y_{i}-\sum_{i=1}^{N} x_{i} \sum_{i=1}^{N} y_{i}}{N \sum_{i=1}^{N} x_{i}^{2}-\sum_{i=1}^{N} x_{i} \sum_{i=1}^{N} x_{i}} .
\end{aligned}
$$

For this case, the dependent variable $y$ corresponds to $r$ and the regressor $x$ to $\delta_{R c}$. The regression coefficients for this interval approximation are computed as

$$
\hat{\beta_{0}}=0,0705^{\circ} \mathrm{s}^{-1} ; \hat{\beta}_{1}=0,0658 \mathrm{~s}^{-1} .
$$

This so-called "global rudder characteristic" shall reproduce a realistic turning behavior in the rudder working range. Since Dieudonné's spiral is a static curve, its interval approximation cannot
Table 3. Nomoto Parameters at Different Nominal Values.

\begin{tabular}{lcc} 
& $\delta_{R c}=0^{\circ}$ & $\delta_{R c}=-4^{\circ}$ \\
\hline$K_{I S}$ in s & 0.1850 & 0.0643 \\
$T_{1}$ in s & 7.3836 & 7.1267 \\
$T_{2}$ in s & 123.1729 & 37.6596 \\
$T_{3}$ in s & 18.0786 & 16.4644 \\
$T_{S}$ in s & 112.4779 & 28.3219 \\
\hline
\end{tabular}

provide the required model parameter. Using engineering judgment, the tangent slope at $\delta_{R c}=-4^{\circ}$ approximates well the slope of the global rudder line. Hence, Eq. (2) are linearized at this nominal value in order to calculate the second set of Nomoto parameters (see Table 3 ).

It must be noted that the slope of the tangent relates to its plant gain so that $K_{I S}$ of parameter set 1 is higher compared to that of set 2 , corresponding to Fig. 4. Assuming an equal system gain, it can be expected that the controller gain for the first set of parameters will be smaller and for the second one higher.

\subsection{PID-controller Design}

This subsection shows the controller design performed separately for each linearized system. For simplicity, Nomoto's 2nd-order model is used for the design process. Priority is given to comparable designs, because both determined $\mathrm{PIDT}_{1^{-}}$ controller are finally applied on the same nonlinear system comparing their performance.

Using the root-locus method, a control system of the course angle is designed restricted to the following performance specifications:

- maximum overshoot $e_{\max } \leq 5 \%$,

- settling time $t_{\epsilon} \leq 90 \mathrm{~s}$,

- settling interval $\epsilon=3 \%$,

- rudder angle $\delta_{R} \leq 15^{\circ}$.

For achieving the desired performance, the root loci are reshaped by inserting a phase-lead compensator into the open loop so that two branches intersect a pair of dominant poles. This pair of dominant complex-conjugate closed-loop poles is placed at the desired location specified by the damping ratio $D$ and the natural frequency $\omega_{0}$ calculated with the specifications.

The transfer functions of the controllers are:

$$
\begin{aligned}
G_{P D T_{1}}(s) & =K_{R}\left(1+\frac{T_{D} s}{1+T s}\right), \\
G_{P I D T_{1}}(s) & =K_{R}\left(1+\frac{1}{T_{I} s}+\frac{T_{D} s}{1+T s}\right),
\end{aligned}
$$

where $K_{R}$ - proportional gain, $T_{D}$ - derivative time constant, $T$ - delay time constant, and $T_{I}-$ integral time constant. 
Table 4. $\mathrm{PDT}_{1}$-Parameter and Performance of Step Response $\Delta \psi_{c}=-5^{\circ}$, Linear System.

\begin{tabular}{lcc} 
& $\delta_{R c}=0^{\circ}$ & $\delta_{R c}=-4^{\circ}$ \\
\hline$K_{R}$ in s & 0.21 & 0.60 \\
$T_{D}$ in s & 112.5 & 15.0 \\
$T$ in s & 11.2 & 1.5 \\
$e_{\max }$ in $\%$ & 3.6 & 3.8 \\
$t_{\epsilon}$ in s & 80.4 & 123.6 \\
$\delta_{R, \max }$ in ${ }^{\circ}$ & -9.3 & -16.0 \\
\hline
\end{tabular}

Table 5. PIDT $_{1}$-Parameter and Performance of Step Response $\Delta \psi_{c}=-5^{\circ}$, Nonlinear System.

\begin{tabular}{lcc} 
& $\delta_{R c}=0^{\circ}$ & $\delta_{R c}=-4^{\circ}$ \\
\hline$K_{R}$ in s & 0.21 & 0.60 \\
$T_{I}$ in s & 150.0 & 429.0 \\
$T_{D}$ in s & 112.5 & 30.0 \\
$T$ in s & 11.2 & 3.0 \\
$e_{\max }$ in $\%$ & 11.3 & 12.8 \\
$t_{\epsilon}$ in s & 494.4 & 311.0 \\
$\delta_{R, \max }$ in & \\
\hline
\end{tabular}

The determined parameters $K_{R}, T_{D}$, and $T$ are used for the PIDT $_{1}$-controller, while the integral time constant $T_{I}$ is derived from the condition that an actuator disturbance input of $\delta_{R c}=1^{\circ}$ shall be vanished within the settling interval in $1000 \mathrm{~s}$ (Fossen, 1994). For both controllers, the I-gain $K_{I}=\frac{K_{R}}{T_{I}}$ is chosen to be equal, because both sets of parameters are determined for the same ship with its asymmetric properties.

In order to compare both controller designs, the most important design criterion is

$$
K_{R, 1} K_{I S, 1}=K_{R, 2} K_{I S, 2},
$$

where the index 1 denotes Nomoto parameter set 1 and index 2 set 2 , respectively (Table 3 and 5 ).

This design process is conducted for both sets of Nomoto parameters. For the first set, the specifications are met for the phase-lead compensator after a course step $\left(\psi_{c}(t>0)=-5^{\circ}\right)$ is applied on the linear system. For the second set, the $\mathrm{PDT}_{1}$ controller cannot achieve the requested rudder angle criterion due to the small derivative time constant in conjunction with the required performance. Therefore, the settling time is increased to $150 \mathrm{~s}$ so that the specifications can now be fulfilled (Table 4). The determined parameters of the PIDT $_{1}$-controller shown in Table 5 meet the criterion of Eq. (9).

\subsection{Comparison of the Course Controllers}

Both developed controllers are compared with course step and ramp functions of the nonlinear plant given in Eq. 2.

For a step of the commanded course angle of $\Delta \psi_{c}=-5^{\circ}$, the responses of the controlled nonlinear system show that the course angle of

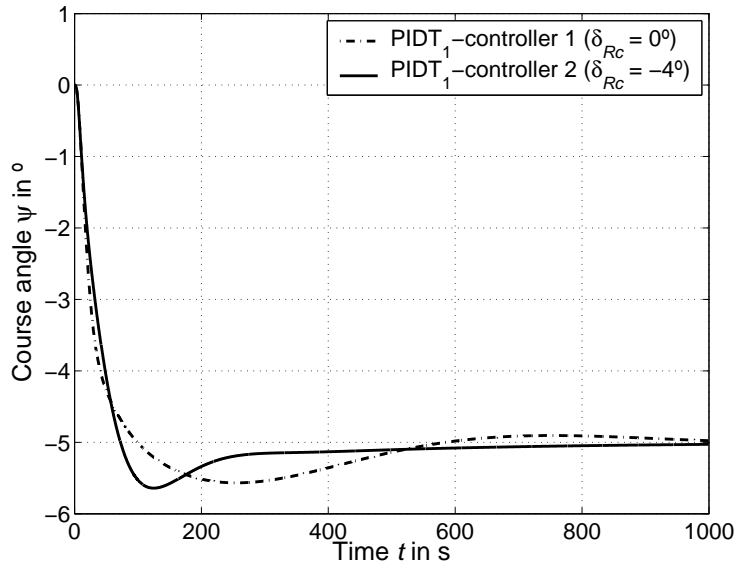

Fig. 5. Step Response of the Nonlinear System $\left(\Delta \psi_{c}=-5^{\circ}\right)$.

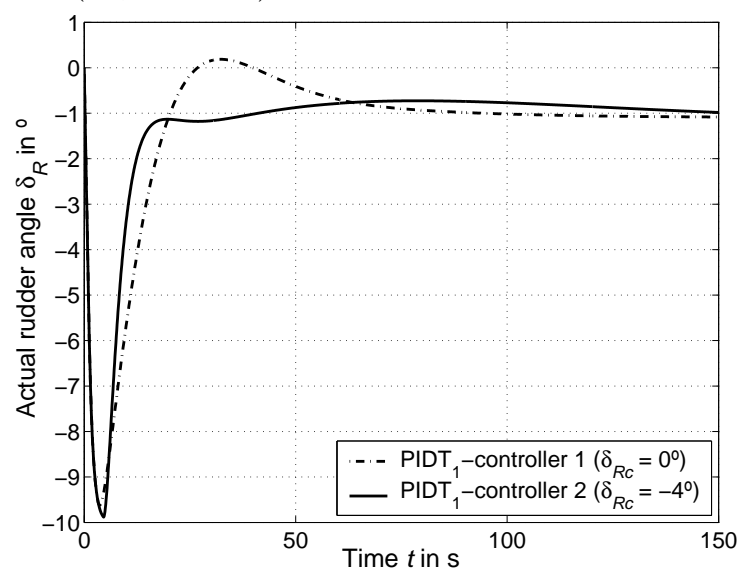

Fig. 6. Corresponding Rudder Angles $\delta_{R}(t)$.

controller $2\left(\delta_{R c}=-4^{\circ}\right)$ is settled in less than two thirds of the time compared to controller 1 , although the overshoot is quite similar, see Fig. 5 and Table 5. It may be noted that the system performance is slower than expected and the specifications of Subsection 3.3 cannot be met. This is caused by the I-element of the controller and rough approximations of the linearized model used for controller design. Figure 6 shows that for the second controller design, the amount of the maximum rudder angle is slightly increased. The early flattening of this rudder angle is probably the result of a high controller gain associated with a higher gain of the nonlinear plant for small rudder angles.

Finally, results of a course alteration maneuver are presented which is chosen for its practical relevance. This maneuver is simulated with a ramp function, $\Delta \psi_{c}=20^{\circ}$ in $t=50 \mathrm{~s}$. Figure 7 shows an approximately identical overshoot for both controllers $(11.3 \%$ vs. $10.0 \%)$ while the commanded course angle is reached clearly faster $(225.0 \mathrm{~s}$ vs. $504.0 \mathrm{~s})$. The plot of the rudder angles shows whether an advantage nor disadvantage (Fig. 8). 


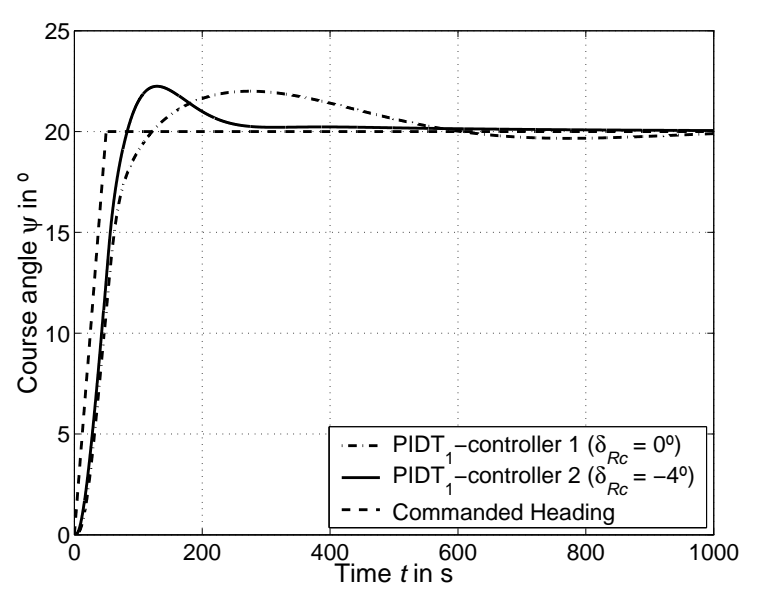

Fig. 7. Ramp Response of the Nonlinear System $\left(\Delta \psi_{c}=20^{\circ}, r=0,4^{\circ} \mathrm{s}^{-1}\right)$.

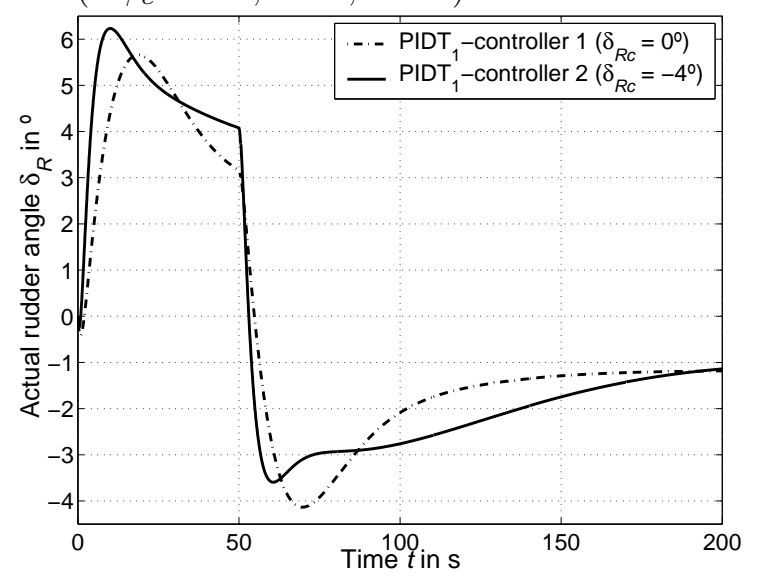

Fig. 8. Corresponding Rudder Angles $\delta_{R}(t)$.

\section{CONCLUSION}

The investigations outlined in this paper aimed at designing a course controller for ships where the turning ability is strongly influenced by nonlinearities of the plant.

Therefore, the linear controller design uses an interval approximation of Dieudonné's spiral in the working range of the rudder to provide better results while applying the controller on the nonlinear course plant. A distinct faster settling of the course angle is observed in conjunction with a similar rudder deflection. This alternative approach results in a more realistic turning ability for a larger rudder range than the conventional Nomoto model.

It is shown that the positive proof of yaw stability is insufficient in applying the Nomoto model linearized at rudder zero to nonlinear plants. The difficulties in identifying the vessel "Transeuropa" support the argument that theoretical examinations even for modern ships are required in practice. Therefore, more hydrodynamic information provided by the ship yard is needed for a suitable controller design.
A possible criteria for recognizing nonlinearities of a plant might be the fact that different results of parameter identifications are obtained for the first part of a course alteration maneuver and a complete one. Thus, additional experimental and theoretical investigations has to be carried out to clarify this issue.

Further fields of investigation lie in a concept of a nonlinear controller design for considerable nonlinearities in spiral curves and adaptive course and track controls with an online parameter identification.

\section{REFERENCES}

Chislett, M. S. and J. Strøm-Tejsen (1965). Planar Motion Mechanism Tests and Full-scale Steering and Manoeuvring Predictions for a Mariner Class Vessel. International Shipbuilding Progress 12(129), pp. 201-224.

Clarke, D., P. Gedling and G. Hine (1982). The Application of Manoeuvring Criteria in Hull Design Linear Theory. Transactions RINA.

Fossen, T. I. (1994). Guidance and Control of Ocean Vehicles. John Wiley \& Sons Ltd. Chichester, England.

Kleinau, D. (1976). Untersuchung zur Steuerbarkeit des Frachtschiffes Typ 301 "Pioner" der Neptunwerft Rostock, Teil II. Schiffbauforschung 15(5/6), pp. 140-151.

Korte, H. et al. (2001). Concept of a Modern Manoeuvre Prediction System for Ships. IFAC CAMS. Glasgow, UK. Paper No. WA 4-3.

Korte, H. et al. (2002). Entwicklung eines Prototypen für ein Manöver-Prädiktions-System für Schiffe mit externem Strömungsmess- und -informationssystem. Abschlussbericht MAPSYS (18 S 0163 A). Universität Rostock.

Lampe, B. P. et al. (1998). Einsatz integrierter Navigationssysteme zur hochgenauen Ortsbestimmung, Bahnplanung und Bahnführung in der Seeschifffahrt (INIS). Abschlussbericht (50 NC 9502/7). Universität Rostock.

Mewis, F. (2001). Reynoldszahleffekte an PodAntrieben. Schlussbericht (18S0146 A). Hamburgische Schiffbau-Versuchsanstalt $\mathrm{GmbH}$ (HSVA). Hamburg, Germany.

Mierau, J.-W. (1983). Ein neues Adaptionsverfahren für Kursregler. Jahrbuch der Schiffbautechnischen Gesellschaft.

Nomoto, K., T. Taguchi, K. Honda and S. Hirano (1957). On the steering qualities of ships. Int'l Shipbuilding Progress 4(35), pp. 354-370.

Schmitz, G. (1961). Anwendung der Theorie des schlanken Körpers auf die dynamische Gierstabilität und Steuerbarkeit von Schiffen. Wissenschaftliche Zeitschrift der Universität Rostock 10(2/3), pp. 175-190. 